\title{
Floristic Diversity of Puliyanamkunnu, Chalavara Grama Panchayath, Palakkad District, Kerala State
}

\author{
Praveenkumar K
}

\author{
PG Department of Botany \& Research Centre, Sree Krishna College, Guruvayur, Ariyannur P. O., Thrissur District, 680102, \\ Kerala, India \\ Email: praveenkumarkvk1992@g mail.com
}

\begin{abstract}
Botanical exploration in Puliyanamkunnu, Chalavara Grama Panchayath of Palakkad district yielded a total of 80 wild taxa of angiosperms. These taxa belong to 65 genera covering 38 families including 36 herbs, 12 climbers, 14 Shrubs and 18 trees. Among them 12 species are endemics and 10 species are rare and red listed. Out of these 80 taxa, 69 species are recorded to be used in different systems of medicines like Ayurveda, Siddha, Unani, Tibetan, Homeopathy, Folk and Western or Modern system. The data will provide information on herbs, Shrubs, climbers and trees that exist in the Puliyanamkunnu, Chalavara Grama Panchayath and about the natural condition under which these plants grow. Such studies will provide reliable information on the presents and distribution of plants in general. This data coupled with studies on threat assessment and trade can also guide focused. Conservation actions like species recovery for each species its local uses are provided. Voucher specimens are deposited in the Sree Krishna college herbarium, with the correct botanical identity and medicinal uses recorded in brief.
\end{abstract}

Keywords-Diversity, Chalavara, Palakkad.

\section{INTRODUCTION}

A botanical exploration was carried out in the Puliyanamkunnu, Chalavara Grama Panchayath, Ottapalam taluk, Palakkad district, Kerala state to collect and study the rare and endemic plants of the region. The Puliyanamkunnu is a hill area located in Puliyanamkunnu water shed near Anangan mala ecotourism project, Palakkad district of Kerala. During the survey, a local guide (Member of Panchayath level Peoples Biodiversity Register Committee) and a medicine man belonging to Mannan community, accompanied us for helping in locating the medicinal plants. Since ages the Mannan and Peruvannan community has been using these plants to treat a number of ailments and diseases. However, due to lack of proper documentation, the knowledge is getting lost. The present paper is an effort to focus attention on the documentation and preservation of the traditional knowledge on some of the endemic, rare, red listed and medicinal plants. This is especially important because the last couple of decades have brought the plantbased medicines back into focus of research as well as development of value added products (Alagesaboopathi \& Balu 1999).

Mannan is the minor scheduled community living in the Palakkad district and their lives and economy are intimately interlinked with the nature. They depend mostly upon the forest flora and fauna for their livelihood. This community collects and utilizes many plants for food, fibre, fuel and medicines and their very survival depends on the non-wood forest produce of the region. The present paper deals with the information gathered from an old Mannan vaidyar and the local healer of the community about the medicinal plants used in the health care of the tribe is given. Eighty such plants are included this paper.

\section{STUDY AREA}

Puliyanamkunnu is a moist deciduous forest element with a low altitude plains area covering three wards of Chalavara Grama Panchayath, Ottapalam taluk, Palakkad district (Champion \& Seth, 1968). The Puliyanamkunnu watershed starting from Pulinchimala and ending in Angadithodu. Two major roads passing through this area, 23 ponds, 2 kanals, 7 sacred groves are there. The present study conducted in the Puliyanamkunnu watershed area has resulted in the collection of 80 taxa of angiosperms coming under 65 genera and 38 families. Out of these, 22 rare, endemic, red listed and taxonomically important species are enumerated here with updated nomenclature, local name, 
family, brief description, distribution and habitat, phenological data, specimen examined and notes for better understanding of these taxa. The voucher specimens are deposited at the Sree Krishna College, Guruvayur.

\section{MATERIALS AND METHODS}

The study area was visited during different seasons and two specimens were collected in each species and these were systematically numbered and tagged. Important field observation like, habit, phenology of the plant, colour, texture and smell of leaves, abundance, local names and local uses available were also noted. Each species in fresh condition was critically studied with the help of floras like, Flora of Presidency of Madras (Gamble, 1915-1936); Flowering Plants of Kerala (Sasidharan, 2004). The plants were identified with the help of floras and finally by comparing with the reference collections available in the Herbarium of Kerala Forest Research Institute, Peechi. The species were often poisoned, processed and labeled, by standard herbarium methods given by Santapau (1955) and Jain \& Rao (1977).

\section{RESULTS AND DISCUSSION}

The present study conducted in the Puliyanamkunnu watershed area has resulted in the collection of 80 taxa of angiosperms. These taxa belong to 65 genera covering 38 families including 36 herbs, 12 climbers, 14 Shrubs and 18 trees. Among them 12 species are endemics and 10 species are rare and red listed. Out of these 80 taxa, 69 species are recorded to be used in different systems of medicines like Ayurveda, Siddha, Unani, Tibetan, Homeopathy, Folk and Western or Modern system. (Ravikumar et al., 2000; Udayan \& Indira Balachndran, 2009).

In the present paper a total of 80 species were collected and they are arranged alphabetically. Information provided includes botanical name, family, habit, systems of medicine and status. (Table 1)

Table.1: List of species collected

A: Ayurveda, F: Folk, H: Homeopathy, M: Western or Modern, S: Siddha, T: Tibetan and U: Unani.

\begin{tabular}{|c|c|c|c|c|c|}
\hline $\begin{array}{l}\text { Sl. } \\
\text { No. }\end{array}$ & Botanical name & Family & Habit & Systems of medicine & Status \\
\hline 1 & Anamirta cocculus (L.) Wight \& Arn. & Menispermaceae & Climber & F,H,M,S,U & Medicinal \\
\hline 2 & Abelmoschus moschatus Medik. & Malvaceae & Herb & $\mathrm{A}, \mathrm{F}, \mathrm{S}, \mathrm{U}$ & Cultivated \\
\hline 3 & Abrus precatorius L. & Fabaceae & Climber & $\mathrm{A}, \mathrm{F}, \mathrm{H}, \mathrm{S}, \mathrm{T}$ & Medicinal \\
\hline 4 & Acalypha indica L. & Euphorbiaceae & Herb & $\mathrm{A}, \mathrm{F}, \mathrm{H}, \mathrm{S}, \mathrm{U}$ & Medicinal \\
\hline 5 & Acanthospermum hispidum DC. & Asteraceae & Herb & - & - \\
\hline 6 & Aegle marmelos (L.) Correa & Rutaceae & Tree & $\mathrm{A}, \mathrm{F}, \mathrm{H}, \mathrm{T}, \mathrm{U}$ & Red listed \\
\hline 7 & Alstonia scholaris (L.) R. Br. & Apocynaceae & Tree & $\mathrm{A}, \mathrm{F}, \mathrm{H}, \mathrm{T}, \mathrm{U}$ & Medicinal \\
\hline 8 & Artocarpus hirsutus Lam. & Moraceae & Tree & $\mathrm{A}, \mathrm{F}$ & Endemic \\
\hline 9 & Alysicarpus vaginalis (L.) DC. & Fabaceae & Herb & $\mathrm{F}$ & Red listed \\
\hline 10 & Bambusa bambos (L.) Voss & Poaceae & Tree & $\mathrm{A}, \mathrm{F}, \mathrm{S}, \mathrm{U}$ & Medicinal \\
\hline 11 & Bougainvillea spectabilis Willd. & Nyctaginaceae & Shrub & $\mathrm{F}$ & Cultivated \\
\hline 12 & Breynia retusa (Dennst.) Alston & Euphorbiaceae & Shrub & $\mathrm{A}, \mathrm{F}, \mathrm{S}$ & Medicinal \\
\hline 13 & Breynia vitis-idaea (Burm. f.) C.E.C. Fisch. & Euphorbiaceae & Shrub & $\mathrm{F}, \mathrm{S}, \mathrm{U}$ & Medicinal \\
\hline 14 & Canscora pauciflora Dalz & Gentianaceae & Herb & - & Red listed \\
\hline 15 & Canthium rheedei DC. & Rubiaceae & shrub & $\mathrm{F}$ & Red listed \\
\hline 16 & Catharanthus pusillus (Murr.) G. Don & Apocynaceae & Herb & $\mathrm{F}, \mathrm{S}$ & Red listed \\
\hline 17 & Curcuma neilgherrensis Wight & Zingiberaceae & Herb & - & Red listed \\
\hline 18 & Cynanchum tunicatum (Retz.) Alston in Trimen & Asclepiadaceae & Climber & - & Red listed \\
\hline 19 & Cycas circinalis $\mathrm{L}$ & Cycadaceae & Tree & $\mathrm{A}, \mathrm{F}, \mathrm{S}, \mathrm{U}$ & Red listed \\
\hline 20 & Dipteracanthus prostratus (Poir.) Nees & Acanthaceae & Herb & $\mathrm{F}$ & Red listed \\
\hline 21 & Drosera burmannii Vahl & Droseraceae & Herb & $\mathrm{F}$ & Red listed \\
\hline 22 & Elephantopus scaber L. & Asteraceae & Herb & $\mathrm{A}, \mathrm{F}, \mathrm{S}, \mathrm{U}$ & Medicinal \\
\hline 23 & Eleusine indica (L.) Gaertn. & Poaceae & Herb & $\mathrm{A}, \mathrm{S}$ & Medicinal \\
\hline 24 & Emilia sonchifolia (L.) DC. & Asteraceae & Herb & $\mathrm{A}, \mathrm{F}, \mathrm{S}, \mathrm{U}$ & Medicinal \\
\hline
\end{tabular}




\begin{tabular}{|c|c|c|c|c|c|}
\hline 25 & Eragrostis viscosa (Retz.) Trin. & Poaceae & Herb & - & - \\
\hline 26 & Ficus tsjahela Burm. f. & Moraceae & Tree & $\mathrm{F}$ & - \\
\hline 27 & Gomphrena celosioides Mart. & Amaranthaceae & Herb & $\mathrm{F}$ & - \\
\hline 28 & Gomphrena globosa L. & Amaranthaceae & Herb & $\mathrm{S}, \mathrm{F}$ & Cultivated \\
\hline 29 & Hemidesmus indicus (L.) R.Br. & Periplocaceae & Climber & $\mathrm{A}, \mathrm{F}, \mathrm{S}, \mathrm{T}, \mathrm{U}$ & Red listed \\
\hline 30 & $\begin{array}{l}\text { Heteropogon contortus (L.) P. Beauv. ex Roem. } \\
\& \text { Schult. }\end{array}$ & Poaceae & Herb & $\mathrm{F}$ & - \\
\hline 31 & Hibiscus hispidissimus Griff. & Malvaceae & Herb & A & - \\
\hline 32 & Holigarna arnottiana Hook. f. & Anacardiaceae & Tree & F,S & Red listed \\
\hline 33 & Holostemma ada-kodien Schult. & Asclepiadaceae & Climber & $\mathrm{A}, \mathrm{F}, \mathrm{U}$ & \\
\hline 34 & Hoppea fastigiata (Griseb.) Clarke & Gentianaceae & Herb & - & Red listed \\
\hline 35 & Hybanthus enneaspermus (L.) F. Muell. & Violaceae & Herb & $\mathrm{A}, \mathrm{F}, \mathrm{S}$ & Medicinal \\
\hline 36 & Hyptis suaveolens (L.) Poit., & Lamiaceae & Herb & $\mathrm{F}$ & - \\
\hline 37 & Impatiens chinensis L. & Bals aminaceae & Herb & $\mathrm{F}$ & - \\
\hline 38 & Indigofera colutea (Burm. f.) Merr. & Fabaceae & Herb & - & - \\
\hline 39 & Indigofera linnaei Ali. & Fabaceae & Herb & $\mathrm{A}, \mathrm{F}, \mathrm{S}$ & Medicinal \\
\hline 40 & Ipomoea hederifolia L. & Convolvulaceae & Climber & $\mathrm{F}$ & Cultivated \\
\hline 41 & Ipomoea obscura (L.) Ker-Gawl. & Convolvulaceae & Climber & $\mathrm{A}, \mathrm{F}, \mathrm{S}$ & Medicinal \\
\hline 42 & Ixora coccinea $L$. & Rubiaceae & Shrub & $\mathrm{A}, \mathrm{F}, \mathrm{S}, \mathrm{U}$ & Medicinal \\
\hline 43 & Jasminum sambac (L.) Ait. & Oleaceae & Climber & $\mathrm{A}, \mathrm{F}, \mathrm{S}, \mathrm{T}, \mathrm{U}$ & Medicinal \\
\hline 44 & Leucas aspera (Willd.) Link & Lamiaceae & Herb & $\mathrm{A}, \mathrm{F}, \mathrm{H}, \mathrm{S}, \mathrm{U}$ & Medicinal \\
\hline 45 & Lindernia ciliata (Colsm.) Pennell & Scrophulariaceae & Herb & $\mathrm{F}$ & - \\
\hline 46 & Macaranga peltata (Roxb.) Muell.-Arg. & Euphorbiaceae & Tree & $\mathrm{F}, \mathrm{S}$ & - \\
\hline 47 & Memecylon umbellatum Burm.f. & Melastomaceae & Tree & $\mathrm{F}$ & Endemic \\
\hline 48 & Moringa pterygosperma Gaertn. & Moringaceae & Tree & $\mathrm{A}, \mathrm{S}, \mathrm{T}, \mathrm{U}$ & Cultivated \\
\hline 49 & Murraya koenigii (L.) Spreng. & Rutaceae & Tree & $\mathrm{A}, \mathrm{F}, \mathrm{S}, \mathrm{U}$ & Cultivated \\
\hline 50 & Mussaenda frondosa $\mathrm{L}$. & Rubiaceae & Tree & $\mathrm{A}, \mathrm{F}, \mathrm{S}, \mathrm{U}$ & Red listed \\
\hline 51 & Murdannia spirata (L.) Brueck. & Commelinaceae & Herb & - & - \\
\hline 52 & Naregamia alata Wight \& Arn. & Meliaceae & Herb & $\mathrm{A}, \mathrm{F}$ & Red listed \\
\hline 53 & Ocimum americanum L. & Lamiaceae & Herb & $\mathrm{A}, \mathrm{F}, \mathrm{H}$ & Medicinal \\
\hline 54 & Olea dioica Roxb. & Oleaceae & Tree & $\mathrm{F}, \mathrm{S}$ & Endemic \\
\hline 55 & Osbeckia muralis Naud. & Melastomaceae & Herb & - & Endemic \\
\hline 56 & Piper longum L. & Piperaceae & Climber & $\mathrm{A}, \mathrm{F}, \mathrm{S}, \mathrm{T}, \mathrm{U}$ & Red listed \\
\hline 57 & Pseudarthria viscida (L.) Wight \& Arn. & Fabaceae & Herb & $\mathrm{A}, \mathrm{S}, \mathrm{U}$ & Red listed \\
\hline 58 & Rhynchoglossum notonianum (Wall.) Burtt & Gesneriaceae & Herb & - & Red listed \\
\hline 59 & Ricinus communis $\mathrm{L}$. & Euphorbiaceae & Shrub & $\mathrm{A}, \mathrm{F}, \mathrm{S}, \mathrm{T}, \mathrm{U}$ & Medicinal \\
\hline 60 & Saraca asoca (Roxb.) de Wilde & Caesalpiniaceae & Tree & $\mathrm{A}, \mathrm{F}, \mathrm{H}, \mathrm{S}, \mathrm{U}$ & Red listed \\
\hline 61 & Sida cordata (Burm. f.) Borss. & Malvaceae & Herb & $\mathrm{A}, \mathrm{F}, \mathrm{S}, \mathrm{U}$ & Medicinal \\
\hline 62 & Sida fryxellii Sivar. \& Pradeep & Malvaceae & Herb & $\mathrm{A}$ & Endemic \\
\hline 63 & Sida mysorensis Wight \& Arn. & Malvaceae & Herb & A & Medicinal \\
\hline
\end{tabular}




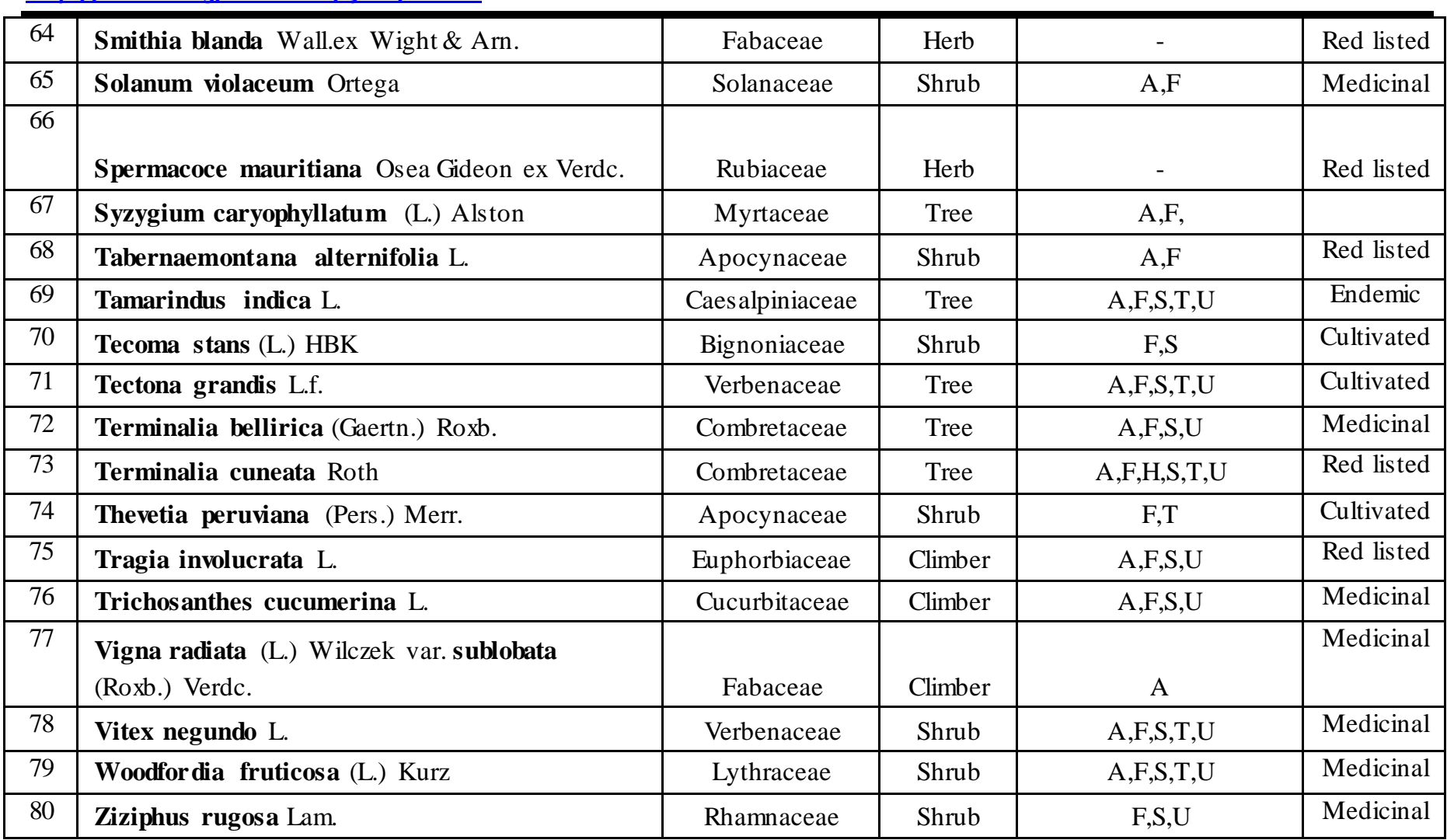

5 medicinal plants were assessed in Conservation Assessment Management Plan Workshop (CAMP) as Red Listed Medicinal Plants were collected from the present study site. Drosera burmanni Vahl (Droseraceae); Holostemmaada-kodien Schult. (Asclepiadaceae); Pseudarthria viscida (L.)Wight \& Arn. (Fabaceae); Trichosanthes cucumerina L. (Cucurbitaceae) and Vigna radiata (L.) Wilczek var. sublobata (Roxb.) Verdc. (Fabaceae). 6 medicinal plants were assessed in Conservation Assessment Management Plan Workshop (CAMP) as Red Listed Medicinal Plants were collected from the present study site. Aegle marmelos (L.) Correa (Rutaceae); Artocarpus hirsutus Lam. (Moraceae); Cycas circinalis L. (Cycadaceae); Piper longum L. (Piperaceae); Saraca asoca (Roxb.) de Wilde (Caesalpiniaceae) and Terminalia cuneata Roth (Combretaceae). 2 species are endemic to Western Ghats Canscora pauciflora Dalz. (Gentianaceae) and Osbeckia muralis Naud. (Melastomaceae). 4 species are endemic to Peninsular India Alysicarpus vaginalis (L.) DC. (Fabaceae); Curcuma neilgherrensis Wight (Zingiberaceae) and Naregamia alata Wight \& Arn. (Meliaceae). 2 species are endemic to India Dipteracanthus prostratus (Poir.) Nees (Acanthaceae) Smithia blanda Wall. ex Wight \&Arn. (Fabaceae). 4 species are endemic to Western Ghats Artocarpus hirsutus
Lam. (Moraceae); Holigarna arnottiana Hook.f. (Anacardiaceae); Mussaenda frondosa L. (Rubiaceae) and Tabernaemontana alternifolia L. (Apocynaceae). 1 species are endemic to Peninsular India Canthium rheedei DC. (Rubiaceae). 9 Species Endemic to India and Sri Lanka Catharanthus pusillus (Murray) G. Don (Apocynaceae), Cynanchum tunicatum (Retz.) Alston (Asclepiadaceae), Hemidesmus indicus (L.) R.Br. (Periplocaceae), Hoppea fastigiata (Griseb) Clarke (Gentianaceae), Polygala rosmarinifolia Wight \&Arn. (Polygalaceae), Pseudarthria viscida (L.) Wight \& Arn. (Fabaceae), Rhynchoglossum notonianum (Wall.) Burtt. (Gesneriaceae), Spermacoce mauritiana Oseagideon ex Verdc. (Rubiaceae) and Tragia involucrata L. (Euphorbiaceae).

\section{CONCLUSION}

Out of 80 plants 69 plants are medicinal. Here endemic plants Artocarpus hirsutus, Memecylon malabaricum, Osbeckia muralis, and Olea dioica are present. When compare to habit of species more number of herbs and shrubs are present, but less numbers of trees. This shows the disturbance inside the natural habitat. In this circumstance suitable management measures and awareness programmes about the importance of sacred groves are necessary for sustainable utilization of the valuable 
bioresources. Chalavara Grama Panchayath proposed suitable plans to protect this area.

\section{ACKNOWLEDGEMENTS}

We are grateful to the Kerala Biodiversity Board, Govt. of Kerala for providing facilities to carry out the study. We are thankful to the members and staffs of Chalavara Grama Panchayath for granting permission to conduct the study and providing information about the area.

\section{REFERENCES}

[1] Alagesaboopathi, C. \& Balu, S. 1999. Ethnobotany of Indian Andrographis Wallich ex Nees. J. Econ. Tax. Bot., 23(1) 29-32.

[2] Champion, H.G. and S.K. Seth. 1968. A Revised Survey of the Forest Types of India. Forest Research Institute, Manager of Publications, Delhi.

[3] Gamble, J.S. and C.E.C. Fischer, 1915-1936. The Flora of the Presidency of Madras. parts 1-11 (parts 17 by Gamble and $8-11$ by Fischer), Vols. 1-3. Adlard\& Sons Ltd., London.

[4] Jain, S.K. and R.R. Rao, 1977. A Handbook of Field and Herbarium Methods. Today \& Tomorrow, New Delhi.

[5] Ravikumar, K. and D.K. Ved. (Assisted by R. Vijaya Sankar \& P.S. Udayan) 2000. Illustrated Field Guide of 100 Red-Listed Medicinal Plants of Conservation Concern in Southern India. FRLHT, Bangalore.

[6] Santapau, H. 1955. Botanical Collector's manual - A Handbook. New Delhi.

[7] Sasidharan, N 2004. Biodiversity documentation for Kerala Part 6 Flowering Plants. Kerala. Forest Research Institute, Peechi, Kerala.

[8] Udayan, P.S. and Indira Balachandran, 2009. Medicinal Plants of Arya Vaidya Sala Herb Garden. Kottakkal Arya Vaidya Sala, Kottakkal. 